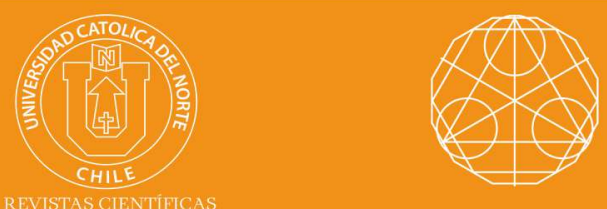

\title{
A new generalization of Wilson's functional equation
}

Hajira Dimou* iD orcid.org/0000-0003-4545-3647

Abdellatif Chahbi** iD orcid.org/0000-0002-1312-5772

Samir Kabbaj $^{* * *}$ (iD orcid.org/0000-0001-9717-9366

*Ibn Tofail University, Dept. of Mathematics, Kenitra, Morocco.

vimouhajira@gmail.com

"* Ibn Tofail University, Dept. of Mathematics, Kenitra, Morocco.

$\square$ abdellatifchahbi@gmail.com

***Ibn Tofail University, Dept. of Mathematics, Kenitra, Morocco.

- samkabbaj@yahoo.fr

Received: September 2018 | Accepted: March 2019

\section{Abstract:}

Let $G$ be a group, let $\sigma: G \rightarrow G$ be an involutive automorphism and let $\chi_{1}, \chi_{2}: G \rightarrow C^{*}$ be two characters of $G$ such that $\chi_{2}(x \sigma(x))=$ 1 for all $x \in G$. The aim of this paper is to describe the solutions $f$, $g: G \rightarrow C$ of the functional equation

$$
\chi_{1}(y) f(x y)+\chi_{2}(y) f(\sigma(y) x)=2 f(x) g(y), x, y \in G,
$$

in terms of characters and additive functions.

Keywords: Wilson's equation; Group; Semigroup involutive automorphism; Multiplicative function.

MSC (2010): 39B52.

\section{Cite this article as (IEEE citation style)}

H. Dimouand, A. Chahbi and S. Kabbaj,"A new generalization of Wilson's functional equation", Proyecciones (Antofagasta On line), vol. 38, no. 5, pp. 943-954, Dec. 2019, doi: 10.22199/issn.0717-6279-2019-05-0060. [Accessed ddmm-yyyy

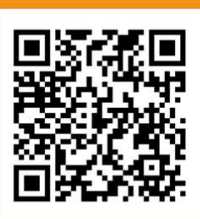

Article copyright: (c) 2019 Hajira Dimou, Abdellatif Chahbi and Samir Kabbaj This is an open access article distributed under the terms of the Creative Commons Licence, which permits unrestricted use and distribution provided the original author and source are credited. 


\section{Notation and terminology}

Throughout the paper we work in the following framework and with the following notation and terminology.

Let $G$ be a group and $S$ be a semigroup (a set with an associative composition rule).

Let $\sigma: G \rightarrow G$ be an involutive automorphism, that is an homomorphism such that $\sigma \circ \sigma=i d$. If $(G ;+)$ is an abelian group, then the inversion $\sigma(x):=-x$ is an example of an involutive automorphism. Another example is the complex conjugation map on the multiplicative group of non-zero complex numbers.

A function $A: G \rightarrow \mathbf{C}$ is called additive, if it satisfies $A(x y)=A(x)+$ $A(y)$ for all $x, y \in G$.

A multiplicative function on $S$ is a map $\mu: S \rightarrow \mathbf{C}$ such that $\mu(x y)=$ $\mu(x) \mu(y)$ for all $x, y \in S$. A character on a group $G$ is a homomorphism from $G$ into the multiplicative group of non-zero complex numbers.

If $S$ is a topological space, then we let $C(S)$ denote the algebra of continuous functions from $S$ into $\mathbf{C}$.

\section{Introduction}

In [13] Stetkær obtained the complex-valued solutions of the following version of d'Alembert's functional equation

$$
f(x y)+\chi(y) f\left(x y^{-1}\right)=2 f(x) f(y), \quad x, y \in G,
$$

where $G$ is a group and $\chi$ is a character of $G$. The non-zero solutions of equation (2.1) are the normalized traces of certain representation of the group $G$ on $\mathbf{C}^{2}$.

In [12], Stetkær obtained the complex-valued solutions of the following variant of d'Alembert's functional equation

$$
f(x y)+f(\sigma(y) x)=2 f(x) f(y), \quad x, y \in S,
$$

where $\sigma$ is an involutive automorphism of a semigroup $S$. The solutions of equation (2.2) are the form $f(x)=\frac{\varphi(x)+\varphi(\sigma(x))}{2}, x \in S$, where $\varphi: S \rightarrow \mathbf{C}$ is multiplicative. In [3], Elqorachi and Redouani extended the result of Stetkær by solving the functional equation

$$
f(x y)+\chi(y) f(\sigma(y) x)=2 f(x) f(y), \quad x, y \in S,
$$


where $\chi: S \rightarrow \mathbf{C}^{*}$ is a multiplicative function of $S$ such that $\chi(x \sigma(x))=1$ for all $x \in S$.

In [15], Wilson's wrote a paper almost a hundred years ago on the group $G=\mathbf{R}$, where they coalesce to the equation

$$
f(x+y)+f(x-y)=2 f(x) g(y), \quad x, y \in \mathbf{R},
$$

which is known in the literature as Wilson's first generalization of d'Alembert's functional equation. For more informations about the functional equation (2.4) see Aczél [1, Section 3.2.1 and Section 3.2.2]. We note that the solutions formulas of equation (2.4) for abelian groups are known.

Wilson's functional equation on a group $G$ takes the form

$$
f(x y)+f\left(x y^{-1}\right)=2 f(x) g(y), \quad x, y \in G,
$$

where $f, g: G \rightarrow \mathbf{C}$ are two unknown functions. In [4] Ebanks and Stetkær studied the solutions $f, g: G \rightarrow \mathbf{C}$ of Wilson's functional equation (2.5) and the following variant of Wilson's functional equation (see [14])

$$
f(x y)+f\left(y^{-1} x\right)=2 f(x) g(y), \quad x, y \in G .
$$

They solve (2.6) and they obtained some new results about (2.5).

The main purpose of this paper is first to solve the functional equation

$$
\chi_{1}(y) f(x y)+\chi_{2}(y) f(\sigma(y) x)=2 f(x) g(y), \quad x, y \in G,
$$

where $\chi_{1}, \chi_{2}: G \rightarrow \mathbf{C}^{*}$ are two characters of the group $G$ such that $\chi_{2}(x \sigma(x))=1$ for all $x \in G$, and second to present several consequences of this result as well. In particular, using our main result, we find the complex-valued solutions of the following functional equations

$$
\begin{gathered}
f(x y)+f(\sigma(y) x)=2 f(x) g(y), \quad x, y \in G . \\
\chi_{1}(y) f(x y)+f(y x)=2 f(x) g(y), \quad x, y \in G . \\
\chi_{1}(y) f(x y)+\chi_{2}(y) f(\sigma(y) x)=2 f(x), \quad x, y \in G .
\end{gathered}
$$

Finally, we note that the sine addition law on semigroups given in $[5,11]$ is a key ingredient of the proof of our main result (Theorem 3.3). 


\section{Solution of equation (2.7)}

In this section we obtain the solutions of the functional equation (2.7) on groups. The following lemma will be used in the proof of Theorem 3.3.

Lemma 3.1. Let $S$ be a semigroup, let $\sigma: S \rightarrow S$ be an involutive automorphism and let $\chi: S \rightarrow \mathbf{C}^{*}$ be a multiplicative function. The solutions $g: S \rightarrow \mathbf{C}$ of the functional equation

$$
g(x y)+\chi(y) g(\sigma(y) x)=2 g(x) g(y), \quad x, y \in S,
$$

are the functions of the form

$$
g=\frac{\mu+\chi \mu \circ \sigma}{2}
$$

where $\mu: S \rightarrow \mathbf{C}$ is a multiplicative function such that

$$
[\chi(x \sigma(x))-1] \mu(x)=0 \quad \text { for all } x \in S .
$$

Moreover, if $S$ is a topological semigroup and $g \in C(S)$, then $\mu, \chi \mu \circ \sigma \in$ $C(S)$

Proof. Making the substitutions $(x y, z),(\sigma(z) x, y)$ and $(x, y z)$ in $(3.1)$, we get respectively

$$
\begin{aligned}
g(x y z)+\chi(z) g(\sigma(z) x y) & =2 g(x y) g(z), \\
g(\sigma(z) x y)+\chi(y) g(\sigma(y z) x) & =2 g(\sigma(z) x) g(y), \\
g(x y z)+\chi(y z) g(\sigma(y z) x) & =2 g(x) g(y z) .
\end{aligned}
$$

Multiplying the middle identity by $\chi(z)$ and using

$$
\chi(z) g(\sigma(z) x)=2 g(x) g(z)-g(x z)
$$

we get

$$
\chi(z) g(\sigma(z) x y)+\chi(y z) g(\sigma(y z) x)=4 g(x) g(y) g(z)-2 g(y) g(x z) .
$$

Subtracting this equation from the sum of equations (3.2) and (3.4) we get after some simplifications that

$$
g(x y z)-g(x) g(y z)=[g(x y)-g(x) g(y)] g(z)+[g(x z)-g(x) g(z)] g(y) .
$$


With the notation $g_{a}(b):=g(a b)-g(a) g(b)$ we can reformulate (3.5) to $\mathrm{g}_{x}(y z)=g_{x}(y) g(z)+g_{x}(z) g(y)$.

This shows that the pair $\left(g_{x}, g\right)$ satisfies the sine addition law for any $x \in S$.

Case 1: Suppose that $g_{x}=0$ for all $x \in S$. By the definition of $g_{x}$, we see that $g$ is multiplicative. Substituting $g$ into (3.1), we infer that $\chi g \circ \sigma=g$. So we are in the form claimed in Lemma 3.1 with $\mu=: g$.

Case 2: We now suppose that $g_{x} \neq 0$ for some $x \in S$. From the known solution of the sine addition law (see for instance [5] or [11, Theorem 4.1]), we see that there exist two multiplicative functions $\chi_{1}, \chi_{2}: S \rightarrow \mathbf{C}$ such that

$$
g=\frac{\chi_{1}+\chi_{2}}{2} .
$$

We may assume that they are different, because we otherwise are back in the multiplicative case already treated. Substituting $g$ into (3.1) we find after a reduction that

$$
\chi_{1}(x)\left[\chi_{2}(y)-\chi(y) \chi_{1} \circ \sigma(y)\right]+\chi_{2}(x)\left[\chi_{1}(y)-\chi(y) \chi_{2} \circ \sigma(y)\right]=0
$$

for all $x, y \in S$. Since $\chi_{1} \neq \chi_{2}$ we get from the theory of multiplicative functions (see for instance [11, Theorem 3.18]) that both terms are 0, so

$$
\left\{\begin{array}{l}
\chi_{1}(x)\left[\chi_{2}(y)-\chi(y) \chi_{1} \circ \sigma(y)\right]=0 \\
\chi_{2}(x)\left[\chi_{1}(y)-\chi(y) \chi_{2} \circ \sigma(y)\right]=0
\end{array}\right.
$$

for all $x, y \in S$. Since $\chi_{1} \neq \chi_{2}$ at least one of $\chi_{1}$ and $\chi_{2}$ is not zero.

Subcase 2.1: Suppose that $\chi_{2}=0$. Hence $\chi_{1} \neq 0$. From (3.6), we see that $\chi \chi_{1} \circ \sigma=\chi_{2}=0$ which implies that $\chi_{1}=0$. But that contradicts $\chi_{1} \neq 0$. So this subcase is void. The same is true for $\chi_{1}=0$ and $\chi_{2} \neq 0$.

Subcase 2.2: We now suppose that $\chi_{1} \neq 0$ and $\chi_{2} \neq 0$. From (3.6), we see that $\chi_{2}(y)=\chi(y) \chi_{1}(\sigma(y))$ and $\chi_{1}(y)=\chi(y) \chi_{2}(\sigma(y))$ for all $y \in S$. So

$$
[\chi(y \sigma(y))-1] \chi_{1}(y)=0 \quad \text { for all } y \in S,
$$

and arrive at the desired form with $\mu=: \chi_{1}$. 
The other direction of the proof is trivial to verify.

The continuity statement follows from [11, Theorem 3.18(d)].

Since a non-zero multiplicative function on a group is a character of this group, then Lemma 3.1 implies the following result.

Corollary 3.2. Let $G$ be a group, let $\sigma: G \rightarrow G$ be an involutive automorphism and let $\chi$ be a character of $G$. The functional equation (3.1) has a non-zero solution $g: G \rightarrow \mathbf{C}$ if and only if $\chi(x \sigma(x))=1$ for all $x \in G$, and in that case $g$ has the form

$$
g=\frac{\mu+\chi \mu \circ \sigma}{2}
$$

where $\mu$ is a character of $G$.

Theorem 3.3. Let $G$ be a group, let $\sigma: G \rightarrow G$ be an involutive automorphism and let $\chi_{1}, \chi_{2}$ be two characters of $G$ such that $\chi_{2}(x \sigma(x))=1$ for all $x \in G$. The solutions $f, g: G \rightarrow \mathbf{C}$ of the functional equation (2.7) are the following:

1. $f=0$ and $g$ is arbitrary.

2. When $\chi_{1}(x \sigma(x))=1$ for all $x \in G$, then $g$ has the form

$$
g=\frac{\mu+\chi_{1} \chi_{2} \mu \circ \sigma}{2}
$$

where $\mu$ is a character of $G$. Furthermore, we have:

1. If $\mu \neq \chi_{1} \chi_{2} \mu \circ \sigma$, then

$$
f=\alpha \chi_{1} \circ \sigma \mu+\beta \chi_{2} \mu \circ \sigma .
$$

for some $(\alpha, \beta) \in \mathbf{C}^{2} \backslash\{(0,0)\}$.

2. If $\mu=\chi_{1} \chi_{2} \mu \circ \sigma$, then there exists an additive function $A: G \rightarrow$ C with $A \circ \sigma=-A$ such that

$$
f=(\alpha+A) \mu \chi_{1} \circ \sigma,
$$

for some $\alpha \in \mathbf{C}$. 
3. When $\chi_{1}(x \sigma(x)) \neq 1$ for some $x \in G$, then the pair $(f, g)$ has the form

$$
g=\frac{\mu+\chi_{1} \chi_{2} \mu \circ \sigma}{2} \text { and } f=\alpha \chi_{2} \mu \circ \sigma,
$$

where $\mu$ is a character of $G$ and $\alpha \in \mathbf{C}^{*}$.

Moreover, if $G$ is a topological group, $f \neq 0$ and $f, g \in C(G)$, then $\mu, \chi_{1} \chi_{2} \mu \circ \sigma, A \in C(G)$

Proof. It is elementary to check that the cases stated in the Theorem define solutions of (2.7), so it is left to show that any solution $f, g: S \rightarrow \mathbf{C}$ of (2.7) falls into one of these cases. If $f=0$, then (1) is the case. From now on we assume that $f \neq 0$ (which implies that $g \neq 0$ ).

Let $x, y, z \in S$ be arbitrary. If we replace $x$ by $x y$ and $y$ by $z$ in (2.7), we get

$$
\chi_{1}(z) f(x y z)+\chi_{2}(z) f(\sigma(z) x y)=2 f(x y) g(z) .
$$

One the other hand, if we replace $x$ by $\sigma(z) x$ in (2.7), we infer that

$$
\chi_{1}(y) f(\sigma(z) x y)+\chi_{2}(y) f(\sigma(y z) x)=2 f(\sigma(z) x) g(y) .
$$

Replacing $y$ by $z$ in (2.7), we obtain

$$
\chi_{1}(z) f(x z)+\chi_{2}(z) f(\sigma(z) x)=2 f(x) g(z),
$$

so by using $\chi_{2}(z \sigma(z))=1$ we have

$\chi_{1}(y) f(\sigma(z) x y)+\chi_{2}(y) f(\sigma(y z) x)=2 \chi_{2}(\sigma(z)) g(y)\left[2 f(x) g(z)-\chi_{1}(z) f(x z)\right]$.

Replacing $y$ by $y z$ in (2.7), we obtain

$$
\chi_{1}(y z) f(x y z)+\chi_{2}(y z) f(\sigma(y z) x)=2 f(x) g(y z) .
$$

Since

$$
\chi_{2}(y) f(\sigma(y z) x)=\chi_{2}(\sigma(z))\left[2 f(x) g(y z)-\chi_{1}(y z) f(x y z)\right],
$$

again using $\chi_{2}(z \sigma(z))=1$ we have

$$
\begin{aligned}
\chi_{2}(z) \chi_{1}(y) f(\sigma(z) x y) & +\left[2 f(x) g(y z)-\chi_{1}(y z) f(x y z)\right] \\
& =2 g(y)\left[2 f(x) g(z)-\chi_{1}(z) f(x z)\right] .
\end{aligned}
$$


Subtracting this from $\chi_{1}(y)$ multiply by equation (3.7), we get after some simplifications that

$\chi_{1}(y z) f(x y z)-f(x) g(y z)=g(y)\left[\chi_{1}(z) f(x z)-f(x) g(z)\right]+g(z)\left[\chi_{1}(y) f(x y)-f(x) g(y)\right]$.

With the notation

$$
f_{x}(y)=\chi_{1}(y) f(x y)-f(x) g(y),
$$

equation (3.14) can be written as follows

$$
f_{x}(y z)=f_{x}(y) g(z)+f_{x}(z) g(y) .
$$

This shows that the pair $\left(f_{x}, g\right)$ satisfies the sine addition law for any $x \in S$. In particular for $x=e$, where $f_{e}=\chi_{1} f-f(e) g$. From this last equality we infer that

$$
f=\frac{1}{\chi_{1}}\left[f(e) g+f_{e}\right]
$$

In the rest of the proof, we discuss the following two cases.

Case 1: Suppose that $f_{e}=0$. Then $f=\frac{f(e)}{\chi_{1}} g$ and hence $f(e) \neq 0$ (because $f \neq 0$ ). By using (2.7) and (3.17), we see that $g$ satisfies the functional equation

$$
g(x y)+\frac{\chi_{2}(y)}{\chi_{1}(\sigma(y))} g(\sigma(y) x)=2 g(x) g(y), \quad x, y \in S .
$$

By using Corollary 3.2 and the fact that $g \neq 0$, we infer that the last equation has a solution if and only if $\chi_{1}(x \sigma(x))=1$ for all $x \in G$ and in that case

$$
g=\frac{\mu+\frac{\chi_{2}}{\chi_{1} \circ \sigma} \mu \circ \sigma}{2}=\frac{\mu+\chi_{1} \chi_{2} \mu \circ \sigma}{2}
$$

where $\mu$ is a character of $G$. So we are in case (2).

Case 2: We now suppose that $f_{e} \neq 0$. From [5, Lemma 3.4], we see that there exist two multiplicative functions $\psi_{1}, \psi_{2}: S \rightarrow \mathbf{C}$ such that

$$
g=\frac{\psi_{1}+\psi_{2}}{2} \text {. }
$$


Subcase 2.1: If $\psi_{1} \neq \psi_{2}$, then $f_{e}=c\left(\psi_{1}-\psi_{2}\right)$ for some constant $c \in$ $\mathbf{C} \backslash\{0\}$. From equality (3.17), we find after a reduction that

$$
f=\frac{1}{\chi_{1}}\left[\alpha \psi_{1}+\beta \psi_{2}\right]
$$

where $\alpha=\frac{f(e)}{2}+c$ and $\beta=f(e)-\alpha$. Substituting $f$ and $g$ in (2.7), we get after some simplifications that

$\alpha \psi_{1}(x)\left[\chi_{2}(y) \psi_{1} \circ \sigma(y)-\chi_{1} \circ \sigma(y) \psi_{2}(y)\right]+\beta \psi_{2}(x)\left[\chi_{2}(y) \psi_{2} \circ \sigma(y)-\chi_{1} \circ \sigma(y) \psi_{1}(y)\right]=0$

for all $x, y \in G$. Since $\psi_{1} \neq \psi_{2}$, we get from the theory of multiplicative functions (see, for instance, [11, Theorem 3.18] that both therms are 0 , so

$$
\left\{\begin{array}{l}
\alpha \psi_{1}(x)\left[\chi_{2}(y) \psi_{1} \circ \sigma(y)-\chi_{1} \circ \sigma(y) \psi_{2}(y)\right]=0 \\
\beta \psi_{2}(x)\left[\chi_{2}(y) \psi_{2} \circ \sigma(y)-\chi_{1} \circ \sigma(y) \psi_{1}(y)\right]=0
\end{array}\right.
$$

for all $x, y \in S$. Since $f \neq 0$ at last one of $\alpha$ and $\beta$ is not zero.

Assume that $\alpha \neq 0$ and $\beta=0$. Then $f=\frac{\alpha}{\chi_{1}} \psi_{1}$ and hence $\psi_{1} \neq 0$ (because $f \neq 0$ ) so that $\psi_{1}$ is a character of $G$. By using (3.18) we infer that $\psi_{2} \neq 0$ and $\chi_{2} \psi_{1} \circ \sigma=\psi_{2} \chi_{1} \circ \sigma$. So $\psi_{2}$ is a character of $G$ and $\psi_{1}=\chi_{1} \chi_{2} \psi_{2} \circ \sigma$ and arrive at the solution in case $(2)(i)$ or case (3) above with $\mu:=\psi_{2}$. We get the same solutions for the case $\alpha=0$ and $\beta \neq 0$.

Assume that $\alpha \neq 0$ and $\beta \neq 0$. For this to be the case we must have $\psi_{1} \neq 0, \psi_{2} \neq 0, \chi_{2} \psi_{1} \circ \sigma=\psi_{2} \chi_{1} \circ \sigma$ and $\chi_{2} \psi_{2} \circ \sigma=\psi_{1} \chi_{1} \circ \sigma$. So that $\psi_{1}, \psi_{2}$ are two characters of $G$ and

$$
\chi_{1}(x \sigma(x))=1 \quad \text { for all } x \in G .
$$

So we are in case $(2)(i)$ above with $\mu:=\psi_{1}$.

Subcase 2.2: If $\psi_{1}=\psi_{2}$, then letting $\mu:=\psi_{1}$ we have $g=\mu \neq 0$. So that $\mu$ is a character of $G$. We get from [5, Lemma 3.4] that there exists a non-zero additive function $A: G \rightarrow \mathbf{C}$ for which $f_{e}=\mu A$. From (3.17), we obtain

$$
f=\frac{1}{\chi_{1}}[f(e)+A] \mu .
$$

Substituting $f$ and $g$ in (2.7), we get after some simplifications that

$[A(y)-\beta]\left(\mu \chi_{1} \circ \sigma\right)(y)+[A \circ \sigma(y)+\beta]\left(\chi_{2} \mu \circ \sigma\right)(y)=0 \quad$ for all $y \in G$, 
where $\beta=f(e)+A\left(x_{0}\right)$ and $x_{0} \in G$ is a fixed element. Using (3.19) and the fact that $A \neq 0$, we find that $\mu_{\chi_{1}} \circ \sigma=\chi_{2} \mu \circ \sigma$ (which implies that $\chi_{1}(x \sigma(x))=1$ for all $\left.x \in G\right)$ and that $A \circ \sigma=-A$. So we are in case (2)(ii).

The continuity statement follows from [11, Theorem 3.18 (d)]. This completes the proof.

\section{Applications}

As immediate consequences of Theorem 3.3, we have the following corollaries.

Corollary 4.1. [6, Theorem3.6] Let $G$ be a group and let $\sigma: G \rightarrow G$ be an involutive automorphism. The solutions $f, g: G \rightarrow \mathbf{C}$ of the functional equation (2.8) are the following:

1. $f=0$ and $g$ is arbitrary.

2. There exists a character $\mu$ of $G$ such that

$$
g=\frac{\mu+\mu \circ \sigma}{2} .
$$

Furthermore, we have:

1. If $\mu \neq \mu \circ \sigma$, then

$$
f=\alpha \mu+\beta \mu \circ \sigma .
$$

for some $(\alpha, \beta) \in \mathbf{C}^{2} \backslash\{(0,0)\}$.

2. If $\mu=\mu \circ \sigma$, then there exists an additive function $A: G \rightarrow \mathbf{C}$ with $A \circ \sigma=-A$ such that

$$
f=(\alpha+A) \mu,
$$

for some $\alpha \in \mathbf{C}$.

Moreover, if $G$ is a topological group, $f \neq 0$ and $f, g \in C(G)$, then $\mu, \mu \circ \sigma, A \in C(G)$. 
Proof. It suffices to take $\chi_{1}=\chi_{2}=1$ in Theorem 3.3.

Corollary 4.2. Let $G$ be a group and let $\chi_{1}$ be a character of $G$. The solutions $f, g: G \rightarrow \mathbf{C}$ of the functional equation (2.9) are the following:

1. $f=0$ and $g$ is arbitrary.

2. There exists a character $\mu$ of $G$ and a constant $\alpha \in \mathbf{C}^{*}$ such that

$$
f=\alpha \mu \text { and } g=\frac{1+\chi_{1}}{2} \mu .
$$

Proof. It suffices to take $\sigma=i d$ in Theorem 3.3.

Corollary 4.3. Let $G$ be a group and let $\sigma: G \rightarrow G$ be an involutive automorphism and let $\chi_{1}, \chi_{2}$ be two characters of $G$ such that $\chi_{2}(x \sigma(x))=$ 1 for all $x \in G$. The solutions $f: G \rightarrow \mathbf{C}$ of the functional equation (2.10) are the following:

1. If $\chi_{1} \chi_{2}=1$, then

$$
f=(\alpha+A) \chi_{2},
$$

where $\alpha \in \mathbf{C}$, and $A: G \rightarrow \mathbf{C}$ is an additive function such that $A \circ \sigma=-A$.

2. If $\chi_{1} \chi_{2} \neq 1$, then $f \equiv 0$ is the only solution of functional equation (2.10).

Proof. The proof follows on putting $g=1$ in Theorem 3.3.

\section{Acknowledgements}

The author would like to express his most sincere gratitude to the reviewer for a number of valuable comments which have led to essential improvement of the paper.

\section{References}

[1] J.Aczel and J. Dhombres, Functional equations in several variables. Cambridge: Cambridge University Press, 1989, doi: 10.1017/CB09781139086578. 
[2] A. Chahbi, B. Fadli and S. Kabbaj, "A generalization of the symmetrized multiplicative Cauchy equation", Acta mathematica hungarica, vol. 149, no. 1, pp.170176, Jun. 2016, doi: 10.1007/s10474-016-0584-3.

[3] E. Elqorachi and A. Redouani, "Solutions and stability of variant of Wilson's functional equation", Proyecciones (Antofagasta, On line), vol. 37, no. 2, pp. 317344, Jun. 2018, doi: 10.4067/S0716-09172018000200317.

[4] B.Ebanks, R. Bruce, H. Stetkær, "OnWilson's functional equations”, Aequationesmathematicae, vol. 89, no. 2,pp. 339-354, Apr. 2015, doi: 10.1007/s00010-014-0287-1.

[5] B. Ebanks, H. Stetkær, "D'Alembert's other functional equation on monoids with an involution", Aequationes mathematicae, vol. 89, no. 1, pp. 187-206, Feb. 2015, doi: 10.1007/s00010-014-0303-5.

[6] B. Fadli, D. Zeglami, and S. Kabbaj, "A variant of Wilsons functional equation", Publicationes mathematicae Debrecen, vol. 87, no. 3-4, pp. 415-427, Oct. 2015, doi: 10.5486/PMD.2015.7243.

[7] P. Kannapan, Functional equations and inequalities with applications, New York, NY: Springer, 2009, doi: 10.1007/978-0-387-89492-8.

[8] P. Kannapan, "A functional equation for the cosine", Canadian mathematical bulletin, vol. 11, no. 3, pp. 495-498, Aug. 1968, doi: 10.4153/CMB-1968-059-8.

[9] P. Kannappan, "The functional equation $f(x y)+f(x y-1)=2 f(x) f(y)$ for groups", Proceedings of the American mathematical society, vol. 19, pp. 69-74, 1968, doi: 10.1090/S0002-9939-1968-0219936-1.

[10] H. Stetkær, "On multiplicative maps”, Semigroup forum, vol 63, no. 3, pp. 466468, Oct. 2001, doi: 10.1007/s002330010077.

[11] H. Stetkær, Functional equations on groups, Singapore, World Scientific Publishing, 2013, doi: 10.1142/8830.

[12] H. Stetkær, "A variant of d'Alembert's functional equation", Aequationes mathematicae, vol. 89, no. 3, pp. 657-662, Jun. 2015, doi: 10.1007/s00010-014-0253-y.

[13] H. Stetkær, "D'Alembert's functional equation on groups", Banach center publications, vol. 99, pp. 173-191, 2013, doi: 10.4064/bc99-0-11.

[14] H.Stetkær, "On a variant of Wilson's functional equation on group”, Aequationesmathematicae, vol.68, no. 3,pp. 160-176, Dec. 2004, doi: 10.1007/s00010-004-2758-2.

[15] W. Wilson, "On certain related functional equations", Bulletin of the American mathematical society, vol. 26, no. 7, pp. 300-312, 1920, doi: 10.1090/S00029904-1920-03310-0.

[16] W.Wilson, "Two general functional equations", Bulletin of the American mathematical society, vol. 31, no. 7, pp. 330-334, 1925, doi: 10.1090/S0002-9904-192504045-8. 